\title{
EL COMPARATIVO EN LAS ANTIGUAS VERSIONES LATINAS DEL GENESIS
}

The author studies the rules of the comparative as it is found in the "Vetus Latina" and "Vulgatan versions of the book of Genesis. He examines all the texts of the two versions to see what relation the different Latin constructions have with the Greek LXX version and the Hebrew text so as to reveal the origin of the constructions and the possible novelty of some of them in Latin.

E1 comparativo latino tiene dos construcciones habituales: $I^{\text {a }}$ ) el ablativo, que es un ablativo de punto de partida ${ }^{1}$, por el que se juzga o compara algo: doctior Petro; $2{ }^{a}$ ) la partícula quam: doctior quam Petrus. Estas dos construcciones, aunque se hayan confundido muchas veces, no son idénticas. I.a construcción con quam sirve para expresar una diferencia entre dos términos desiguales. El ablativo se usa para formular un juicio sobre la semejanza entre dos conceptos ${ }^{2}$.

El complemento del comparativo siguió, a grandes rasgos, las siguientes etapas. En el período arcaico era más frecuente el uso de quam que el del ablativo comparativo, pues éste estaba circunscrito a unos pocos tipos de fórmulas fijas ${ }^{3}$. En la época clásica se extiende un poco más el número de estas fórmulas, pero sin superar la media docena el número de estos giros nuevos. A partir de la época augústea se dibujan dos tendencias: una, tradicionalista, representada por Virgilio y Tácito, que se atiene al uso clásico; otra, helenizante, representada por Horacio y Ovidio, que usa libremente el ablativo comparativo ${ }^{4}$.

1 Cf. Leumann-Hofmann-Szantyr, Lateinische Grammatik II, Munich, 1965, p. 107 § 75; A. Ernout-F. Thomas, Syntaxe latine, Paris, 1964, p. 168; B. Mörland Der lateinische Komparationskasus und dessen Ersatz, Oslo, 1933; A. Traglla, Valore e uso dell' ablativo latino di comparazione, Roma, 1947.

2 Cf. M. Bassols, Sintaxis histórica de la lengua latina I, Barcelona, I945, p. 430; A. Lìnout-I. Thomas, o. c., p. 168.

3 Leumann-Hoimann-Szantyr, o. c., p. I08; II. Bassols, o. c., p. 427 s.; M. Bassols, Sintaxis latina I, Madrid, 1963, p. 125.

4 M. Bassols, Sintaxis histórica (o. c.), p. 430; M. Bassols, Sintaxis latina (o. c.), p. 125 s.; Leumann-Hofmant-Szantyr, o. c., p. 108 s.

6 
Junto a estas dos formas habituales de expresar el régimen del comparativo, han existido a través de la latinidad otras varias, originadas sobre todo a partir de la época clásica, consistentes en la sustitución del ablativo comparativo por otros casos como el genitivo ${ }^{1}$ y el dativo ${ }^{2}$, o en el reemplazo del ablativo de comparación y de la partícula quam por otros giros con preposición, como $a b$, prae, ante, praeter, super, supra, inter, extra, ultra, etc. ${ }^{3}$.

En el presente trabajo vamos a examinar el régimen del comparativo en el Génesis de la Vetus Latina (= VL) y de la Vulgata (= Vg), analizando en cuanto nos sea posible todos los textos, para ver la relación que puedan tener las distintas construcciones con la versión griega de los Setenta $(=\mathrm{LXX})$ y con el texto masorético (= 'TM), así como la relación de las versiones latinas entre sí, y la novedad que eventualmente puedan significar en latín algunas de estas construcciones.

Antes de pasar al análisis de los textos, conviene decir unas palabras sobre la construcción del comparativo en hebreo, ya que las antiguas versiones de la Biblia se remontan directamente (la Vulgata) o indirectamente, a través del griego (la Vetus Latina), a la lengua hebrea.

El hebreo - como el arameo bíblico- no sólo carece de formas específicas de comparativo orgánico, como nuestros comparativos mayor, menor, etc., sino que ni siquiera posee los adverbios comparativos de sentido general 'más', 'menos's. Para expresar el comparativo en una frase de dos miembros, el hebreo utiliza la preposición min puesta delante del segundo niembro, perınaneciendo invariable en su estructura el adjetivo, siempre en grado positivo. La preposición min expresa primariamente la idea de punto de partida, separación o alejamiento. De esta acepción se deriva el sentido de diferencia, que es el que tiene $\min$ en las comparaciones ${ }^{5}$. De esta idea - -a diferencia de»- se pasa fácilmente a la de comparación, "más que».

El mismo tipo de construcción con min utiliza el hebreo con verbos de estado, que encierran una idea adjetival, por ejemplo, "ser grandes, "ser pequeño», "ser alto», etc., e incluso también con numerosos verbos,

1 Leumaun-Hofmann-Szantyr, o. c., p. 112 § 75 e; M. Bassols, Sintaxis histórica (o. c.), p. 434 S.; A. Erwout-F. Thomas, o. c., p. I7r.

2 Leumann-Hofmann-Szantyr, o. c., p. $113 \S 75$ f; M. Bassols, Sintaxis histórica (o. c.), p. 435 s.; A. Ernout-F. Thomas, o. c., p. r7x s.

3 Leumann-Hofmann-Szantyr, o. c., p. IxI $\$ 75$ d; M. Bassols, Sintaxis histórica (o. c.), p. 436 ss.; A. Ernout-F. Thomas, o. c., p. r7r.

- P. Joüon, Grammaire de l'hébreus biblique, Paris 2 1947, p. 435 § I $_{4} \mathrm{I}$ g.

s. C. P. Joüon, o. c., p. $406 \S \mathrm{r} 33$ e. 
de estado y activos, que expresall una acción, pero que encierran virtualmente una idea adjetival, por ejemplo, "amar", "complacerse en", "honrar", etc. ${ }^{1}$.

Estas observaciones son importantes para comprender muchas de las construcciones que vamos a analizar a continuación y que a primera vista pudieran dar la impresión de que no se trata de verdaderas construcciones comparativas. No hay que olvidar que se trata de textos de traducción, cuyo original en último término está redactado en lengua hebrea.

Pasamos ya al estudio de los textos. Salvo error u omisión, hemos encontrado en el Génesis I7 textos con min que tienen en hebreo un sentido comparativo. De estos $I 7$ textos la Vg traduce 13 por un comparativo con su correspondiente régimen, omitiendo la construcción comparativa 4 veces $^{2}$. La VL, en cambio, traduce los I7 textos por un comparativo con su correspondiente régimen. Consideradas todas las variantes de estos I7 pasajes, tanto de $1 \mathrm{a} \mathrm{Vg} \mathrm{-} \mathrm{poco} \mathrm{frecuentes-,} \mathrm{como}$ de la VL - muy frecuentes-, tenemos en el Génesis los siguientes regímenes del comparativo.

r. Construcción con ablativo sin preposición.

El número total de veces que aparece esta construcción son $I$; ; de ellas, 9 corresponien a la VL y 8 a la Vg. Los textos son:

I.-Gen. 3, I: prudentior omnibus bestiis (VLvar). astutior ceteris pecoribus (VLrar). callidior cunctis animantibus (Vg).

2.-Gen. 26, I6: possibilior nobis (VL). potentior nobis (Vgvar).

3.-Gen. 32, II: minor sum omni misericordia tua (VLvar). minor sum cunctis miserationibus tuis (Vg).

2 Cf. P. Joüon, o. c., p. 436 ₹ $44 \mathrm{r}$ h.

2 Los textos son: Gen. 29, 30: amorem sequentis priori praetulit; Gen. 34, I9: et ipse erat inclytus in omni domo patris sui; Gen. 4I, 40: uno tantusm regni solio te praecedam; Gen. 43, 34: maiorque autem pars uenit Beniamin ita ut quinque partibus excederet. 
4.-Gen. 34, I9: dignitosior omnibus (VL).

5.-Gen. 37, 3: plus omnibus filiis (VLvar).

6.-Gen. 37, 4: plus reliquis jiliis (VL). plus cunctis filiis $(\mathrm{Vg})$.

7.-Gen. 38, 26: iistior me cst (Vg).

8.-Gen. 48, x9: maior illo erit (VL). maior illo erit $(\mathrm{Vg})$.

9.-Gen. 49, I2: pulchriores uino (Vg). candidiores lacte (Vg). candidiores lacte (VLvar).

Respecto a este grupo de textos cabe observar lo siguiente: aunque el número de pasajes, en una y otra versión, con ablativo sin preposición, es casi idéntico, no se trata, como puede verse, de los mismos textos. Ya dijimos antes que es un tipo de construcción del comparativo completamente normal, atestiguado a través de toda la latinidad $\mathrm{y}$, por tanto, desde este punto de vista no ofrece novedad alguna, a no ser su presencia mayoritaria con respecto a otras construcciones en la VL, versión popular de la Riblia, hecha sin pretensiones literarias, y su presencia también mayoritaria en la $\mathrm{Vg}$, versión con intenciones literarias bien conocidas. El texto del Génesis de la VL nos demuestra que el ablativo comparativo seguía siendo de uso popular, a pesar de la concurrencia que sufría por parte de otros giros. Esto mismo demuestra también, al menos en parte - por la parte que tiene de popular- la Vg, pues de un número total de $\mathrm{I}_{3}$ textos, la $\mathrm{Vg}$ lo emplea 8 veces.

Comparando estos pasajes con los correspondientes de los LXX, constatamos que los traducen dos veces por comparativo y genitivo ${ }^{1}$, otras dos veces por superlativo y genitivo ${ }^{2}$-aunque en estos dos pasajes hay también variantes con comparativo y genitivo-, otras dos veces por un positivo con ómó -en donde se puede detectar un calco hebreo ${ }^{3}-$ y tres veces con verbos, seguidos de (Gen. 37, 3) y દ̌k $($ Gen. 37, 4). En ninguno de estos 9 pasajes puede

1 Los textos son: Gen. 26, 16; 49, 19.

2 Los textos son: Gen. 3, I; 34, I9.

3 Se trata de Gen. 32, II; 49, 12. 
probarse un influjo directo del griego sobre las versiones latinas. Incluso habría que decir que son más exactas y correctas las versiones latinas que la griega, pues no han traducido en ningún caso el positivo hebreo por un superlativo, como to ha hecho la versión griega en dos ocasiones (Gen. 3, I; 34, I9).

\section{Construcción con "quam» detrás de un comparativo}

Número total de veces con variantes: 8 . De éstas, 6 corresponden a la VL y 2 a la Vg. He aquí los textos:

I.-Gen. 4, I3: maior mea causa est quam ut relinquar (VLvar). maior causa est mea quan ut dimittar (VLvar). maius est peccatum meum quam ut remittatur (VLvar). maior est iniquitas mea quam ut ueniam merear $(\mathrm{Vg})$.

2.-Gen. 29, I9: melius est me dare cam tibi quam dare eam alio uiro (VL).

melius est ut tibi ean dem quan uiro alteri (Vg).

3.-Gen. 38, 26: iustior est illa quam ego (VL).

4.-Gon. 49, I2: candidiores dentes eitus quam lac (VLvar).

Ya dijimos antes que se trata de una construcción normal del comparativo. Respecto a la mayor o menor dependencia del griego, baste decir que en Gen. 4, I3 la VL tiene una lección dependiente directa-

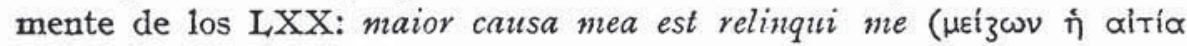

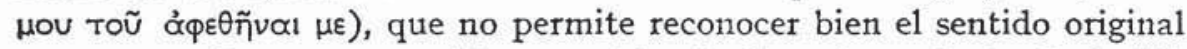
y que reproducen, en cambio, muy bien la Vg y la variante de la VL citada en tercer lugar. La lección de la VL de Gen. 29, I9 es una traducción literal del griego. Los demás textos de este grupo no dependen necesariamiente del griego, sino de las propias estructuras de la irase latina.

\section{Construcción con "quant» detrás de un positivo}

Este tipo de construcción se registra en latín desde la época arcaica y consiste en emplear un positivo en lugar de un comparativo. En estos 
casos, el quam está en lugar de magis quam, potius quam, plus quam. Este uso es frecuente, sobre todo, en los escritores de tendencias arcaicas (Apuleyo, Frontón) y más aún en los escritores vulgares (Quirón). Los escritores eclesiásticos, a partir de Tertuliano, suprimen regularmente el magis, potius, plus, delante de quam, por influjo del griego bíblico ${ }^{1}$.

En el Génesis hay un solo ejemplo como variante de la VL: candidi dentes eius quam lac (Gen. 49, I2). Pero este mismo texto ofrece en la VL la lección: candidi dentes eius magis quam lac, además de otras varias, como veremos más adelante. Los textos abundan en los libros de la actual Vulgata no traducidos por Jerónimo, por ejemplo, Eccli. 20, I: bonum est arguere quam irasci; 16,4: utile mori sine filiis quam relinquere filios impios; 25, 23: commorari leoni et draconi placebit quam habitare cum mulicre nequam; 37, I8: anima uiri sancti enuntiat aliquando uera quan septem circunspectores, etc.

Pero también se encuentran en los libros de la VL revisados por Jerónimo y que forman parte de la actual Vulgata, por ejemplo: Mat. I8, 8: bonum tibi... quam duas manus..., y texto paralelo de Mar. 9, 42; Mat. 18, 9: bonum tibi... quam duos oculos..., y texto paralelo de Mar. 9, 46; Mar. 9, 44: bonum tibi... quam duos pedes...; Luc. 15, 7: gaudium erit... super uno peccatore... quam super nonaginta nouem...; Mat. 5, 29: expedit enim tibi ut pereat unum... quam totım corpus...; y la misma expresión en Mat. 5, 30; I Cor. I4, I9: uolo quinque uerba... quam decem millia uerborum...

Jerónimo, en cambio, en los libros que traduce directamente del hebreo intenta evitar este giro. Es interesante a este respecto la comparación entre el texto del Salmo II7 (II8), 8, 9 del Salterio Galicano revisado por Jerónimo (Vulgata) y su propia traducción del hebreo; en la revisión conserva el giro, en su traducción lo evita:

verso 8: bonum est confidere... quam confidere... (Vg); melius est sperare... quam spcrare... (He);

verso 9: bonum est sperare... quam sperare... (Vg); melius est sperare... quam sperare... (He) ${ }^{2}$.

1 Cf. Leumann-Hofmann-Szantyr, o. c., p. $593 \S 320$ a.

2 H. de Sainte-Marie, Sancti Hieronymi Psalterium iuxta Hebraeos, Roma, 1954, p. I7I. Textos de autores eclesiásticos pueden verse en Leumann-HofmannSzantyr, o. c., p. 594; H. Rönsch, Itala und Vulgata, Marburgo, 1875 (reimpresión, Munich, 1965), p. $44^{2} \mathrm{~s}$. 
La difusión de esta construcción en las versiones latinas de la Biblia depende sin duda del griego; pero su difusión en el griego bíblico depende a su vez del hebreo; se trata, pues, de un calco sintáctico hebreo, al menos en cuanto a su mayor frecuencia ${ }^{1}$.

\section{Construcción con "magis quam" ("plus quam")}

A pesar de lo que hemos dicho en el punto anterior sobre la construcción con quam en vez de magis quam, esta segunda sigue utilizándose con profusión en las versiones latinas de la Biblia. En el Génesis la encontramos 4 veces en la VL y ninguna en la $\mathrm{Vg}$. Los textos son:

I.-Gen. 29, 30: diligebat autem Rebeccam plus quam Liam (VL) ${ }^{2}$.

2.-Gen. 37, 3: Israhel dilexit Ioseph plus quam ceteros filios (VL,var).

3.-Gen. 38, 26: iustificata est Thamar magis quam ego (VL).

4.-Gen. 49, I2: candidi dentes eius magis quam lac (VL).

Respecto a estos textos advertimos que el núm. I es traducción literal del griego: plus quam: $\mu \tilde{\alpha} \lambda \lambda$ ov $\eta_{\eta} . \mathrm{La} \mathrm{Vg}$, en cambio, traduce mucho más libremente el hebreo, evitando la frase comparativa: amorem sequentis priori praetulit. El plus quam del núm. 2 no corresponde literalmente

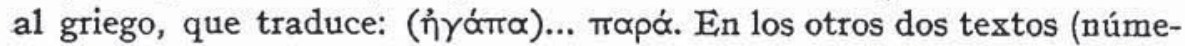
ros 3 y 4) el traductor latino transforma el nै griego en magis quam, evitando el simple quam, que es el modo más frecuente de traducir la partícula griega (véase el punto 3 de nuestra exposición). Por lo demás, la VL tiene en estos dos pasajes otras variantes. Así, en el núm. 3, frente

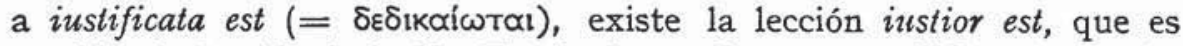

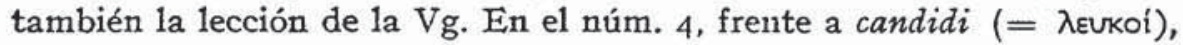
existe también la lección: candidiores; y frente a magis quam $(=\tilde{\eta})$,

1 Cf. M. Zerwick, Graecitas biblica, Roma ${ }_{3}$ 1955, p. 43; F. Blass-A. Debrunner, Grammatik des neutestamentlichen Griechisch, Göttingen ${ }_{12} 1965, \S$ 245; U. Rapallo, "Per una definizione diacronica e tipologica dei calchi ebraici nelle antiche versioni del Levitico", Atti dell' Istituto Lombardo (Rend. Lett.) 103, 1969, p. 380 s.

2 Vetus Latina. Die Reste der altlateinischen Bibel nach Petrus Sabatier new gessanmelt und herausgegeben von der Erzabtei Beuron 2, Genesis, herausgegeben von B. Fischer, Freiburg, 1951, p. 563 (suplementos): aqui se corrige la lección magis quasn por plus quam. 
existe también la variante quam, además de otras varias, como veremos más adelante.

Aunque la Vulgata no tiene ningún ejemplo en el Génesis de la construcción que estudiamos, los tiene, y muy numerosos, en otros libros. Véanse, por ejemplo, los textos siguientes: 2 Sam. 6, 21; Prou. 18, 24; Eccle, 7, I7; 7, 7, 24-25; Is. 54, I; I Sam. 2, 29, etc. El hecho no representa ninguna novedad.

\section{Construcción con genitivo}

El uso del genitivo como caso de comparación aparece en la lengua latina desde la época de Tiberio, siendo el ejemplo seguro más antiguo el texto de Vitrubio 5, I, 3: superiora inferion Se encuentra también en las inscripciones vulgares de esta misma época; luego en Apuleyo (Met. 3, II, 6) y en los juristas contemporáneos (Escélova, Papiniano).

Se discute si es un grecismo o un vulgarismo ${ }^{2}$. Lo que no puede dudarse es que su diiusión en la VL depende del griego. En el Génesis aparece 4 veces, de las cuales 3 corresponden a la VL, y una a la Vg. He aquí los textos:

I.--Gen. 3, I: sapientior omnium (VL).

2.-Gen. 26, I6: poientior nostri (Vg).

3.-Gen. 48, I9: maior illius (VLvar). maior huius (VLvar).

E1 texto de Gen. 3, I presenta en la VL una variedad verdaderamente sorprendente de lecciones:

sapientior omnium bestiarun;

1 Cf. Leumann-Hofmann-Szantyr, o. c., p. II3 $\$ 75$ e. Véanse aquí más autores y textos.

2 Lo consideran un grecismo, entre otros, E. Löfstedt, Syntactica II, Lund, 1933, p. 425, y otros autores en la nota 2; además, M. Bassols, Sintaxis histórica (o. c.), p. 434 s. (por influjo griego); A. Ernout-F. Thomas, o. c., p. 17 I (construcción griega). Lo consideran un vulgarismo, entre otros, Leumann-HofmannSzantyr, o. c., p. II 3 §5 e. 
prudentior omnibus bestiis;

astutior ceteris pecoribus;

sapientissimus cunctis animalibus;

prudentissimus super omnes bestias ${ }^{1}$. tibus.

La Vg, en cambio, ofrece un único texto: callidior cunctis animan-

Prescindiendo ahora de los distintos adjetivos, sapiens, prudens, astutus, para traducir el hebreo 'arūm y de los distintos sustantivos, bestiae, pecora, animalia, para traducir el hebreo hayyah, interesa subrayar aquí los diversos regímenes de los comparativos y superlativos utilizados por los traductores.

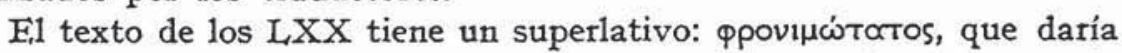
en latín: sapientissimus o prudentissimus omnium, traducción que de hecho se encuentra abundantemente representada en los comentarios de los autores eclesiásticos antiguos ${ }^{2}$. El régimen del superlativo en ablativo: sapientissimus cunctis animalibus, es propio del latín tardío y depende, por una parte, de la confusión entre el comparativo y el superlativo a causa del debilitamiento de su significado $y$, por otra, de la contaminación con las estructuras partitivas ${ }^{3}$.

Las variantes de la VL, en ablativo, lo mismo que el texto de la Vg, se insertan en el grupo de textos que hemos analizado en el apartado I y sobre ellos no hay nada especial que decir. La traducción de la VI: sapientior omnium depende probablemente de la variante griega: фpovt$\mu \omega \dot{t \varepsilon p o s} \pi \alpha ́ v \tau \omega \nu$, en el caso de que el traductor latino la haya conocido. Del texto griego: $\mu \varepsilon i z \omega \nu$ aủroũ dependen sin duda las dos variantes: maior illius y maior huius de Gen. 48, in (VL). La Vg, en cambio, y la recensión L - forma general de la Vetus Latina - presentan aquí el régimen normal en ablativo: maior illo.

E1 texto de la Vg: potentior nostri (Gen. 26, I6), corresponde al griego:

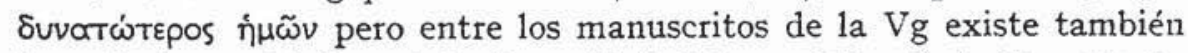
la variante en ablativo: potentior nobis ${ }^{4}$. Este genitivo de la $\mathrm{Vg}$ es tanto más llamativo en cuanto que, por una parte, la VI, tiene aquf precisamente un ablativo: possibilior nobis, y la variante: magnus super nos ${ }^{5}$,

1 Vetus Latina (o. c.), p. 56 .

2 Véanse numerosos textos de autores eclesiásticos en Vetus Latina (o. c.), p. 56 .

3 Cf. Leumann-Hofmann-Szantyr, o. c., p. III $\$ 75$ c.

- Biblia Sacra iuxta Latinam Vulgatam uersionem..., vol. I, Genesis ex interpretatione Sancti Hieronymi, Roma, 1926, p. 255.

5 Vetus Latina (o. c.), p. 276 . 
y, por otra, porque Jerónimo suele evitar en la Vg el régimen en genitivo. Frente a I6 ejemplos, por lo menos, en genitivo en la VL, en la Vg sólo se encuentran 4: Gen. 26, I6 (nuestro texto); Job I2, 3: inferior uestri; Job 13, 2: inferior uestri; Prou. 3, I4: melior auri primi et purissimi ${ }^{1}$.

La conducta de Jerónimo, sin embargo, no deja de ser sorprendente. $\mathrm{Si}$ en $\mathrm{la} \mathrm{Vg}$, es decir, en los libros traducidos por él directamente del hebreo, suele evitar el régimen con genitivo, lo conserva, en cambio, en los libros que revisó y que han pasado a la $\mathrm{Vg}$, por ejemplo, en los Salmos: Ps. 34 (35), Io: fortiorum eius ${ }^{2}$; Ps. 89 (90), ro: amplius eorum ${ }^{3}$; y en el Nuevo Testamento: Mar. 12, 3r: maius hornm; Ioh. 14, 12: maiora horum; Act. I7, II: nobiliores eorum; Hebr. 3, 3: honorem ampliorem domus; Hebr. 6, r6: maiorem sui; 3 Ioh. 4: maiorem horum.

La difusión del genitivo como régimen del comparativo en la VL se debe sin duda a influencia griega, prescindiendo de que esta construcción sea un grecismo o un vulgarismo. Su presencia relativamente frecuente ell la Vg se debe quizá al influjo de la VL y a la difusión que había adquirido esta construcción entre los escritores eclesiásticos 4 .

\section{Construcción con dativo}

Este tipo de construcción tuvo en latín un ámbito muy restringido, originándose al principio en frases como nulli inferior (desde Salustio), nulli secundus (desde Virgilio y Ovidio), adjetivos que siguen la analogía de impar, y que luego se extendieron a otros adjetivos, como ceteris princeps (Apul., Flor. I9), etc. El giro adquiere una cierta frecuencia en el latín tardío: Sidonio, Gregorio de Tours, Venancio Fortunato

1 El número de veces (I6) lo tomamos de Leumann-Hofmann-Szantyr, o. c., p. II 3 $75 \mathrm{e}$, sin responder de su exactitud. El número de veces de la Vg (3), que dan estos mismos autores en la página citada ( $a .$. hat Hieronymus in der Vulgata nur 3 Fälles), hay que ampliarlo con uno más, Prou. 3, r4.

2 Comparativo con genitivo en griego. En su Salterio iuxta Hebraeos Jerónimo traduce: a ualidiore, omitiendo el pronombre; cf. H. de Sainte-Marie, o. c., p. 50 .

3 Comparativo con genitivo en griego. Jerónimo en su Salterio iuxta Hebraeos traduce: quod amplius est; cf. H. de Sainte-Marie, o. c., p. r3x. Este texto no es un comparativo en hebreo.

- Véanse las referencias a autores eclesiásticos y tardios en Leumann-Hofmann-Szantyr, o. c., p. II3 $§ 75 \mathrm{e}$. 
Oribasio y las Vitae patrum, pasando incluso a las lenguas románicas 'inferior a' 1 .

En el Génesis aparece una sola vez en la VL y sólo como variante: maior illi (Gen. 48, I9), en concurrencia con maior illius y maior huius (VL,var), frente al texto recibido: maior illo (VL) y maior illo (Vg).

\section{Construcción con preposiciones}

La sustitución del ablativo comparativo por giros con preposición es de origen post-clásico y adquiere gran desarrollo en el latín tardío, coincidiendo con la tendencia de la lengua a sustituir los casos por giros con preposición. Los regínienes con preposición que aparecen en el Génesis son los siguientes:

\section{a) La preposición "ab»}

Respecto a los textos que vamos a estudiar a continuación hay que advertir dos cosas: primera, que todos ellos se encuentran en la VL. La Vg no presenta ningún ejemplo en el Génesis; pero esto no quiere decir que Jerónimo haya ignorado este giro o lo haya evitado siempre en otros libros bíblicos, como vamos a ver a continuación. Segunda, que hay que distinguir dos tipos de construcción con $a b$ : $a b$ con un comparativo y $a b$ con un positivo.

๙) $A b$ después de un comparativo:

Este giro se remonta a Ovidio, Her. 15, 98: a te dignior, y Plinio, NH XVIII ז26: praestantior $a b$ iis, aunque fuera de los autores eclesiásticos, no se hace general hasta el siglo $\mathrm{IV}^{2}$.

En el Génesis sólo aparece una vez y como variante de la VL: candidiores sunt dentes eins a lacte (Gen. 49, I2). La construcción en este caso

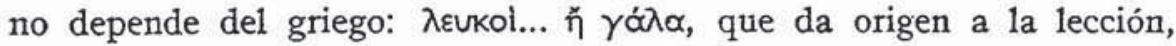
también de la VL, candidi... quam lac, sino que depende del propio uso latino, favorecido por el texto hebreo.

La construcción es frecuente en la $\mathrm{VL}^{3}$. Pero también se encuentra

1 Cf. M. Bassols, Sintaxis histórica (o. c.), p. 435 s.; Leumann-HofmannSzantyr, o. c., p. II 3 \$ 75 f; A. Ernout-F. Thomas, o. c., p. I7I s.

2 Cf. Lcumann-Hofmann-Szantyr, o. c., p. III $\S 75$ d; M. Bassols, Sintaxis histórica (o. c.), p. 437 s.; A. Ernout-F. Thomas, o. c., p. I7I.

3 Véanse otros textos en H. Rönsch, o. c., p. $45^{2}$ s.; U. Rapallo, l. c., p. $37^{8}$ ss. 
en la Vg, por ejemplo, sublimius a solio (I Reg. I, 37); amplius a stulto (Eccle. 6, 8); mclius a filiis (Is. 56, 5); sceleratins ab eis (Ez. I6, 52) ${ }^{1}$. $\mathrm{Y}$ también abunda en los textos de la VL revisados por Jeróniino y que han pasado a formar parte de la Vg, como, nihil minus a magnis apostolis (2 Cor. II, 5); nihil minus ab iis (2 Cor. I2, II); paulo minus ab angelis (Hebr. 2, $7=$ Ps. 8,6 ), etc. ${ }^{2}$. Aparece incluso como variante en el Salterio de Jerónimo inxta Hebraeos, por ejemplo, molliores ab oleo (Ps. 54 [55], 22); mirabilior a me (Ps. 138 [139], 5) ${ }^{3}$. Esto demuestra que Jerónimo no se separa mucho del lenguaje eclesiástico de sus predecesores y contemporáneos 4 .

$\beta) ~ « A b$ después de un positivo:

Ėsta construcción es un hebraísmo que entra en latín con la VL. En el Génesis podemos anotar los siguientes ejemplos

I.-Gen. 3, I4: maledictus tu ab omni pecore (VL). maledictus tu ab omnibus pecoribus (VLvar).

2.-Gen. 32, II: sufficit mihi ab omni iustitia (VL,var). idoneus es mihi ab omni iustitia (VLvar).

3.-Gen. 49, I2: formidolosi oculi eius a uino. fulgentes oculi eins a uino. fului oculi eius a uino. hilares oculi eius a uino. gratiosi oculi eius a uino. gratifici oculi eius a uino. laetifici oculi eius a uino. suffusi oculi eius a uino.

candidi sunt dentes eius a lacte.

1 Cf. H. Rönsch, o. c., p. 452 s.; F. Kaulen, Sprachliches Handbuch zur biblischen Vulgata, Freiburg. 1904 (reimpresión, Hildesheim, 1973), p. 258 s.; W. E. Plater-H. J. White, A grammar of the Vulgate, Oxford, 1926, p. 21.

2 Cf. H. Rönsch, o. c., p. $45^{2}$.

3 Cf. H. de Sainte-Marie, o. c., pp. 80 y 196.

- Usa esta construcción en sus escritos exegéticos y en sus traducciones de la Biblia; en sus cartas, en cambio, sólo aparece tres veces, según Leumann-Hofmann-Szantyr, o. c., p. III $\$ 75$ d. 
Comenzando el análisis por los textos del núm. 3, debemos decir que llama la atención la claridad y uniformidad del texto de la Vg: pulchriores oculi eius uino et dentes eius lacte candidiores, frente a la variedad verdaderamente sorprendente de las lecciones variantes de la VL, variedad que en la primera parte del verso se reduce, como es fácil observar, a los distintos adjetivos usados, sin cambiar en ningún caso el régimen de ablativo con $a b$. En la segunda parte del verso, en cambio, la variedad de regímenes supera ya toda previsión conservando siempre el mismo adjetivo, en positivo, candidi; en comparativo, candidiores. He aquí esas variantes:

a) Con positivo:

\author{
candidi magis quam lac. \\ candidi quam lac. \\ candidi tamquam lac. \\ candidi super lac. \\ candidi a lacte.
}

b) Con comparativo:

\author{
candidiores lacte. \\ candidiores super lac. \\ candidiores tamquam lac. \\ candidiores a lacte.
}

$\mathrm{Si}$ comparamos estas variantes con el texto griego, tenemos en la primera parte del verso un xapotroi... ámò que origina la construcción latina con $a b$ (a vino). Esta misma construcción griega da origen a los

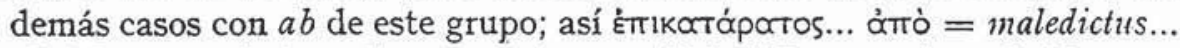
$a b$ (Gen. 3, I4); íavoũta1... ómò = sufficit (idoneus es)... ab (Gen. 32, rI) ${ }^{1}$. Ahora bien, el uso del positivo por el comparativo y la introducción del segundo término de la comparación precedido por la preposición ámò es un calco sintáctico liebreo ${ }^{2}$. Ėn la VL podemos hablar de propagación de calco. Las variantes sufficil (idoneus es)... ab no dan

1 En Gen. 49, 12, sólo el ms. B tiene ùmèp, todos los demás mss. tienen ớrò. Corregir, por consiguiente, el dato de Leumamu-Hofmann-Szantyr, o. c., p. I I 2 , que aluden a ùrrèp como única lección.

2 Cf. M. Zerwick, o. c., p. 43; F. Blass-A. Debrunner, o. c., pp. rig y r53; U. Rapallo, l. c., p. $3^{80}$. 
realmente el sentido original ni expresan con claridad el comparativo que encierran. La Vg y la lección hexaplar O de la VI, indican con claridad que se trata de un comparativo: minor sum omni iustitia.

Por lo que respecta a la segunda parte del verso (Gen. 49, I2), vemos que los traductores latinos han encontrado dificultades en reproducir el $\lambda \varepsilon u k o i . . . \eta \eta n$, originándose así esa variedad sorprendente de lecciones ${ }^{1}$.

E1 régimen con $a b$ después de un positivo es frecuente en la VL. Algunos otros ejemplos serían: humilis a cute (Leu. 13, 4); infima a corio (Leis. 13, 25, 26); infirmus ab (Leu. 13, 21); a mari abundauit cogitatio eius et consilium eius ab abysso magna (Eccli. 24, 39).

Jerónimo suele evitar en la Vg este tipo de construcción, que sin duda le parecía un calco demasiado literal del hebreo; pero no lo descarta del todo. Lo conserva, por ejemplo, en Ez. 6, I4: faciam terrant desolatam et destitutam a deserto Deblatha, 'una tierra más desolada. y devastada que el desierto de Dibla'; Is. 55 , 9: sicut exaltantur caeli a terra, sic exaltatae sunt uiae meae a uiis vestris et cogitationes meae a cogitationibus uestris: 'como son más altos los cielos que la tierra, así son más altos mis caminos que vuestros caminos y mis pensamientos que vuestros pensamientos'. Lo conserva también, y con relativa frecuencia, en los textos que revisó de acuerdo con el griego, por ejeniplo: Ps. 4, 8: dedisti laetitiam in corde meo a fructu frumenti... multiplicati sunt, "has dado a mi corazón más alegría que cuando ellos abundan en el producto del trigo...'; Ps. I7 (I8), 49: ab insurgentibus in me exaltabis me, 'me exaltarás más que (se exaltan) mis agresores'; Ps. 92 (93), 4: a uocibus aquarum multarum mirabiles elationes maris, mirabilis in altis Dominus, 'las ondas del mar son más imponentes (admirables) que los ruidos de aguas inmensas, más imponente (admirable) es el Señor en las alturas'. Adviértase que Jerónimo, en su Salterio inxta Hebraeos, conserva esta misma construcción ${ }^{2}$; en cambio, en el Ps. 4, 8 transforma la frase, dándole otro sentido: in tempore frumentum et uimum eorum multiplicata sunt ${ }^{3}$. La construcción aparece también en el Nuevo Testamento revisado por Jerónimo: descendit hic... iustificatus ab illo (Luc. I8, If) 'bajó éste... más justificado que aqućl'.

Fuera de las versiones bíblicas y de los autores eclesiásticos dependientes de ellas, el giro sólo aparece esporádicamente a partir del s. $\mathrm{v}^{4}$.

1 Algunas de estas lecciones se explican por la variante griega ís que aparece en algunos manuscritos.

2 Cf. H. de Sainte-Marie, o. c., pp. 28 (Ps, 18, 49) y 135 (Ps. 93, 4).

3 H. de Sainte-Marie, o. c., p. I2; algún ms. tiene a tempore.

- Por ejemplo, Hip., Aer. 5: salutares ab iis; Celio Aurel., Chron. 2, 12, ${ }_{3} 8$ : fluorem difficile curabilem... ab eo qui eruptione fuerit effectus; cf. Leumann-Hofmann-Szantyr, o. c., pp. 112 y I70; E. Löfstedt, Syntactica I, Lund, 1942, p. 329 s. 


\section{b) La preposición "super»}

La construcción con super reviste también dos modalidades, con sus características propias. La preposición puede ir detrás de un comparativo o detrás de un positivo. En ambos casos, se trata en último análisis de un hebraísmo, como es también un hebraísmo el uso de vimép en griego para expresar el comparativo ${ }^{1}$. El uso de super como sustitutivo del comparativo empieza en la VL y continúa en la Vg y de aquí pasa a los autores eclesiásticos.

๔) Super después de un comparativo:

Hay dos ejemplos en el Génesis, uno de la VL y otro de la Vg.

I.-Gen. 7, 20: altior fuit aqua super (omnes) montes (Vg).

2.-Gen. 49, I2: candidiores sunt dentes eius super lac (VLvar).

El uso de super detrás de un comparativo origina expresiones pleonásticas de fuerte sabor popular, pues la idea comparativa se expresa dos veces, una, a través del comparativo, y otra, a través de la preposición. Asf́, dentes candidiores super lac, equivale literalmente a 'dientes más blancos sobre la leche', y altior aqua super (omnes) montes, equivale a 'agua más alta sobre (todos) los inontes'.

Debemos ser̃alar, además, que en el uso del comparativo y de la preposición super en los dos textos citados no ha influido para nada el texto griego, ya que en ninguno de los dos aparece la preposición

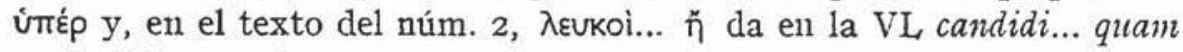
o candidi... magis quain y no candidiores... super.

ß) Super detrás de un positivo:

En el Génesis encontramos 5 ejemplos, 4 de la VI, y uno de la Vg. Los textos son:

x.-Gen. 7, 20: superauit ${ }^{2}$ aqua super excelsos montes (VL).

1 Cf. Leumann-Hofmann-Szantyr, o. c., p. II2 § 75 d; M. Bassols, Sintaxis histórica (o. c.), p. 439; M. Zerwick, o. c., p. 43; F. Blass-A. Debrunner, o. c., $\S 185,3$.

- Presenta, además, otras dos variantes: supercrenit y transcendit, cf. Vetus Latina (o. c.), p. I16. 
2.-Gen. 26, I6: magnus fachus es super nos (VLvar).

3.-Gen. 37, 3: dilexit Ioseph super omnes filios suos (VL). dilegebat Ioseph super omnes filios suos (Vg).

4.-Gen. 49, I2: candidi sunt dentes einls super lac (VLvar).

En este tipo de construcción abundan mucho, en las versiones biblicas, los giros formados por verbos, de estado o de acción, que encierran virtualmente una idea adjetival. E1 uso de super detrás de un positivo corresponde más literalmente al hebreo, ya que en la lengua hebrea, el positivo, que no cambia, hace el oficio de comparativo. Para dar sentido comparativo a un positivo, la lengua latina dispone del recurso a la preposición super, que significa 'sobre', 'encima', 'más allá de', sin necesidad de hallarse en un contexto comparativo. En los textos del Génesis que hemos citado no ha influido la versión griega, pues en ninguno de ellos aparece ímép. La construcción con super detrás de un positivo parece más frecuente que la otra detrás de un comparativo ${ }^{1}$.

\section{c) La preposición "prae»}

En la construcción con prae hay que distinguir dos casos: prae dependiendo de un positivo, y prae dependiendo de un comparativo.

๔) Prae detrás de un comparativo:

Este tipo de construcción, frecuente en el lenguaje popular, es pleonástica, ya que la idea comparativa "1nás que» se expresa dos veces, una con el comparativo, por ejemplo, beatior = 'más feliz', $y$ otra, a través de la preposición, por ejemplo, prae uobis =- 'más que vosotros'.

E1 giro se encuentra ya en Plauto, Epid. 522: me minoris facio prae illo. Lo rehúyeu los autores clásicos; pero aparece de una manera frecuente y sin limitaciones en los autores de tendencias arcaizantes, como Frontón, Apuleyo, Gelio, ${ }^{2}$.

1 Véanse, por ejemplo, de la Vulgata: Leus. I8, II: nagnus super omnes; 2 Sam. I, 26: anabilis super anorem mulierum; Ier. 46, 23: multiplicati sunt super lacustas; Eccle. 7, 20: sapientia confortauit sapientem super decem principes ciuitatis; otros ejemplos del Salterio Galicano, revisado por Jerónimo (= Vulgata): Ps. 17 (18), i8: confortati sunt super me; Ps. I8 (19), II: desiderabilia super aurum; Ps. 50 (5I), 9: super niuem dealbabor, etc.

2 Cf. Leumann-Iloimann-Szantyr, o. c., p. $112 \S 75$ d; M. Bassols, Sintaxis histórica (o. c.), p. $43^{8} \mathrm{~s}$. 
En el Génesis hay un solo ejemplo en la VL: maior pars erat Beniamin... prae illontm partes (Gen. 43, 34), y ninguno en la Vg; pero la construcción es frecuente en otros libros bíblicos, así, por ejemplo, en Dan. r, I5: unltus meliores et corpulentiores prae omnibus pueris; Hebr. I, 4: differentius prae illis nomen haeveditauit; Hebr. 3, 3: amplioris gloriae... prae Moyse, etc.

B) "Prae» detrás de un positivo:

Fista construcción aparece ya en Plauto, Mil. Ir40: non sum dignns prae te, y continúa usándose con mayor o menor frecuencia a través de toda la latinidad ${ }^{1}$.

En el Génesis encontramos dos ejemplos en la VL, como variantes del texto citado antes:

Gen. 43, 34: magnificata facta est pars prae partibus omnium... magnificata facta est pars prae omnium partibus... quam...

Jerónimo emplea este giro en la $\mathrm{Vg}$, por ejemplo, inclytus prae fratribus suis ( 1 Cor. 4,9 ) y en los textos de la VL que revisó, por ejemplo, Ps. 44 (45), 3: speciosus forma prae filiis homimum; Ps. 44 (45), 8: unxit te Deus... prae consortibus tuis, y hasta en su Salterio intia Hebraeos, por ejemplo, Ps. 44 (45), 8: unxit te Deus... prae participibus tuis; Ps. 34 (I35), 5: magnus... Deus noster prae onnibus diis ${ }^{2}$.

d) La preposición "ex»

La construcción con $e x$ para expresar el régimen del comparativo es realmente esporádica. Según el Thesaurus V, 2, II25, 70. aparece desde el P's.-Tert., Huer. 3: eo quod... robustior ex ceteris fuerit, luego en Pelagio, Casiodoro y Oribasio.

El Thesaurus cita el texto del Ps. 138 (139), 6: mirabilis facta est scientia twa ex me, pero no alude al Gen. 32, II de la VL: satis est milit ex omni iustitia et ex omni ueritate. Fin ambos casos se trata de un comparativo, como puede verse por la traducción que dan la Vg y la VI, del texto del Génesis: minor sum cunctis miserationibus (tuis) et ueritate (Vg); minor sum omni misericordia tua et omni veritate (VL), y por

\footnotetext{
1 Leumann-Hofnann-Szantyr, o. c., p. II 2 \$ 75 d; M. Bassols, Sintaxis histórica (o. c.), p. $43^{8}$.

3 H. de Sainte-Marie, o. c., pp. 67 y rgr.

7
} 
las variantes del Salterio de Jerónimo iuxia Hebraeos: mirabilior est scientia tua a me y mirabilior est scientia tua me, por lo que respecta al texto del salmo ${ }^{1}$.

\section{e) La preposición inter}

Este tipo de construcción habría comenzado, según Leumann ${ }^{2}$, con Claudiano Mamerto (s. V) y Casiodoro. Por nuestra parte señalamos un ejemplo en Gen. 3, $\mathrm{I} 4$ (Vg): maledictus es inter omnia animantia, texto que la VL traduce con el ablativo con $a b$ : maledictus $t u$ ab omni pecore.

La construcción con inter, de todos modos, puede prestarse a confusión, ya que en hebreo un positivo puede estar por un comparativo o por un superlativo, bajo ciertas condiciones, cuando se trata de una colectividad $^{3}$, y también en latín un positivo puede hacer las veces de un comparativo o de un superlativo ${ }^{4}$.

Dadas estas posibilidades, el texto que analizamos podría equivaler a un superlativo: 'el más maldito de todos', o a un comparativo: 'maldito más que todos'. La VL lo entendió sin duda en el segundo sentido, y éste es de hecho el sentido que tiene en hebreo, ya que no se dan las circunstancias para que se sobreentienda un superlativo. Sentido comparativo tiene también la frase: benedicta inter mulieres Iahel (Iud. 5, 24), 'Jael, bendita más que las mujeres que habitan en tiendas', 1o mismo que ésta: benedictus eris inter ommes populos, 'serás bendito más que todos los pueblos', etc.

Antes de terminar el análisis de los textos, conviene decir una palabra sobre Gen. 4, II, que presenta un texto oscuro. La VL traduce: maledictus tu a terra quae aperuit os suum. La semejanza con Gen. 3, I4: maledictus $t u$ ab omni pecore (VL) no puede negarse. En griego ambos

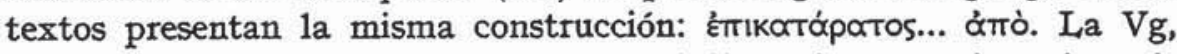
en cambio, tiene inter en un caso: maledictus inter omnia animantia (Gen. 3, I4) y super en el otro: maledictus super terram (Gen. 4, II). La construcción hebrea es idéntica en ambos casos. Pero mientras min en Gen. 3, I4 tiene sentido comparativo, no lo tiene en Gen. 4, II. Ia traducción española sería: 'maldito seas, lejos de esta tierra que abrió su boca...'. La maldición divina contra Caín consiste, en parte, en ser

1 H. de Sainte-Marie, o. c., p. I96.

2 Leumann-Hofmann-Szantyr, o. c., p. $112 \S 75 \mathrm{~d}$.

- Cf. P. Joüon, o. c., p. 437 § 4 I j.

- Cf. Leumann-Hofmann-Szantyr, o. c., p. $169 \S$ roo d; p. $593 \S 320$. 
arrojado 'lejos del país' donde cometió su crimen. Los traductores de 1a VL sintieron dificultades para reproducir el min hebreo, y por eso, además de a terra, existen las variantes in facie terrae y super terram.

Llegados al final de nuestro trabajo, podemos establecer las siguientes conclusiones:

I) E1 régimen más frecuente del comparativo en el Génesis de las antiguas versiones latinas de la Biblia sigue siendo, como en la época clásica, el ablativo sin preposición (I7 ejemplos), la partícula quam con un adjetivo en grado comparativo (8 ejemplos) y la fórmula magis (plus) quam (4 ejemplos).

2) Le sigue luego en importancia, al menos por el número de casos, la construcción con $a b$ detrás de un positivo ( 13 ejemplos), aunque casi todos los ejemplos son variantes de un mismo texto.

3) Las restantes construcciones están representadas por los siguientes ejemplos: genitivo, 4 ; dativo, I; quam con positivo, $\mathrm{I}$; $a b$ con comparativo, I; super con comparativo, 2; super con positivo, 5; prae con comparativo, I; prae con positivo, 2; ex, I; inter, I.

4) La comparación entre la VL y la Vg arroja los siguientes resultados: $1 \mathrm{a} \mathrm{Vg}$ presenta 6 tipos de construcción: ablativo comparativo, quam con comparativo, genitivo, super con comparativo, super con positivo e inter. La VL, en cambio, utiliza I3 tipos de construcción, 5 de ellos comunes con los de la Vg, es decir, todos los citados antes, menos inter, que es propio de la Vg, y los 8 restantes propios, que son: qunill con positivo, magis (plus) quam, dativo, $a b$ con comparativo, $a b$ con positivo, prae con comparativo, prae con positivo y ex.

5) Respecto al origen de las diversas contrucciones, podemos señalar lo siguiente:

a) La construcción con genitivo puede ser de origen vulgar, pero su presencia en el Génesis se debe a la influencia de la versión griega de los LXX.

b) La construcción con super, en cualquiera de sus modalidades, es decir, con positivo o comparativo, es, en último análisis, un hebraísmo.

c) La construcción con quam y positivo, en sustitución de magis (plus) quam, en los textos bíblicos se debe también a influencia de la versión griega de los LXX.

d) La construcción con $a b$ y positivo es un hebraísmo que ha pasado a la VL a través del griego.

e) La construcción con prae detrás de un comparativo tiene origen popular. 
f) La construcción con ex, muy esporádica, en la VL depende del griego.

6) La variedad de construcciones en la VL, depende de la variedad de estas mismas construcciones en la versión griega de los $\mathrm{LXX}$, y esta variedad se debe a la dificultad de traducir la partícula comparativa hebrea min. La Vg evita muchos de estos grecismos o vulgarismos de la VL.

O. García de la Fuente 